\title{
Adaptive Integral Observer-Based Synchronization for Chaotic Systems with Unknown Parameters and Disturbances
}

\author{
Xiuchun Li, ${ }^{1}$ Jianhua Gu, ${ }^{1}$ and Wei $X u^{2}$ \\ ${ }^{1}$ Centre for High Performance Computing, Northwestern Polytechnical University, Xian 710072, China \\ ${ }^{2}$ Department of Applied Mathematics, Northwestern Polytechnical University, Xian 710072, China
}

Correspondence should be addressed to Xiuchun Li; lixiuchun@nwpu.edu.cn

Received 15 January 2013; Accepted 21 April 2013

Academic Editor: J. Liang

Copyright (C) 2013 Xiuchun Li et al. This is an open access article distributed under the Creative Commons Attribution License, which permits unrestricted use, distribution, and reproduction in any medium, provided the original work is properly cited.

\begin{abstract}
Considering the effects of external perturbations on the state vector and the output of the original system, this paper proposes a new adaptive integral observer method to deal with chaos synchronization between the drive and response systems with unknown parameters. The analysis and proof are given by means of the Lyapunov stability theorem and Barbalat lemma. This approach has fewer constraints because many parameters related to chaotic system can be unknown, as shown in the paper. Numerical simulations are performed in the end and the results show that the proposed method is not only suitable to the representative chaotic systems but also applied to some neural network chaotic systems.
\end{abstract}

\section{Introduction}

The introduction and successful application of master-slave method proposed by Pecora and Carroll [1] have inspired the people's passion for researching chaos synchronization. Up to now, people pay more attention to such synchronization scheme that all states of the system are often assumed to be totally known. Common methods are active-passive control [2], adaptive control [3-5], impulsive control [6, 7], sliding mode control [8-10], and so forth. In fact, only partial state information is available in many practical systems. Therefore, applying the observer-based theory to chaos synchronization is very important.

Based on the observer design technique, many observerbased synchronization schemes [11-18] have been proposed. References [11-13] have made chaotic system with known parameters and without external perturbations achieve synchronization based on the observer method. In practice, exotic disturbances exist inevitably. So in $[14,15]$, the chaotic systems with external perturbations have been discussed in order to realize chaos synchronization, but all parameters of the systems $[14,15]$ are supposed to be totally known. Sometimes, the parameters of chaotic systems cannot be known in advance. Aimed at this, References $[16,17]$ have researched such dynamical systems with unknown parameter and external perturbations using adaptive observer, but the papers in $[16,17]$ discuss the effects of external disturbances on the state variables and do not consider the effects of external disturbances on the outputs of the system. When the output has disturbances, the gain matrix will enlarge the effect of the output disturbances using traditional observer, and then the so-called integral observer is proposed [18]. Reference [18] has implemented integral observer theory and the nonlinear approximation ability of the orthogonal neural networks to realize chaos synchronization, where the gain matrix has been obtained using Linear Matrix Inequality (LMI) technique, but the proposed method [18] needs to know the bounds of external disturbances, and, the paper in [18] only consider the effect of external disturbances on the output of the system and do not discuss the effect of external disturbances on the state vector of the system.

Based on the above-mentioned problems, we consider the effect of external perturbations not only on the state vector but also on the output of the original system; adaptive integral observer controller is designed for chaos synchronization with unknown parameters. From the discussion in Section 3, we can see that the proposed scheme has fewer constraints, where the bounded disturbance and other parameters related 
to chaotic system can be unknown. Finally, the Rössler chaotic system and cellular neural network chaotic system are illustrated to verify the effectiveness and feasibility of the method.

The rest of this paper is structured as follows. We start with system description in Section 2. Then, method of design of adaptive integral observer and its proof are given in Section 3. Numerical simulations are shown in Section 4. And Section 5 gives some conclusions of this paper.

\section{System Description}

In a common secure communication system, the message is injected onto a chaotic carrier in transmitter. To recover the message in the receiver, synchronization of the transmitter and the receiver systems is crucial. When the state vector and the output are perturbed due to some reason, a class of chaotic dynamical systems can be described in the following equations:

$$
\begin{gathered}
\dot{x}=A x+f(x)+B g(x) \theta+d(t), \\
y=C x+D_{2}(t),
\end{gathered}
$$

where $x \in R^{n}$ is an $n$-dimensional state vector of the system (1), $y \in R^{m}$ is a $p$-dimensional output vector of the system (1), $A \in R^{n \times n}, B \in R^{n \times p}$, and $C \in R^{m \times n}$ are all known constant matrices, $\theta \in R^{q}$ is unknown parameter vector of chaotic system (1), $d(t) \in R^{n}$ and $D_{2}(t) \in R^{m}$ are external perturbations of the state and the output vector, respectively, and $f: R^{n} \rightarrow R^{n}$ and $g: R^{n} \rightarrow R^{p \times q}$ are nonlinear functions of chaotic system (1).

System (1) represents a class of chaotic dynamical systems. It includes not only the representative chaotic systems, such as Lorenz system, Rőssler system, and Chua's system, but also some neural network systems, such as cellular neural network (CNN) system and Winner-Take-All competed neural network system.

It regards (1) as the drive system; the response system is

$$
\dot{\hat{x}}=A \widehat{x}+f(\widehat{x})+B g(\widehat{x}) \hat{\theta}+K(y-C \widehat{x}),
$$

where $K$ is gain matrix.

Now the synchronization error between the drive and response systems is defined as $e=x-\widehat{x}$, and then the error system is obtained

$$
\begin{aligned}
\dot{e}= & (A-K C) e+f(x)-f(\widehat{x}) \\
& +B(g(x) \theta-g(\widehat{x}) \hat{\theta})+d(t)-K D_{2}(t) .
\end{aligned}
$$

It is easily found that the error system cannot achieve asymptotic stability due to existence of the perturbation $K D_{2}(t)$. Furthermore, the larger gain $K$ causes more influence of the perturbation $K D_{2}(t)$ on the error system because of multiplicative relationship.
So, we propose a new method that is adaptive integral observer to make the perturbed chaotic system realize synchronization. The drive system (1) is rewritten as

$$
\begin{gathered}
\dot{x}=A x+f(x)+B g(x) \theta+d(t), \\
\dot{z}=C x+D_{2}(t), \\
y=C x+D_{2}(t),
\end{gathered}
$$

where the vector $z=\int_{0}^{t} y(\xi) d \xi$, that is, a definite integral of the original output $y$.

Suppose that $X=\left(\begin{array}{l}x \\ z\end{array}\right), \widehat{A}=\left(\begin{array}{ll}A & 0 \\ C & 0\end{array}\right), \widehat{B}=\left(\begin{array}{l}B \\ 0\end{array}\right), D_{1}(t)=$ $\left(\begin{array}{c}d(t) \\ 0\end{array}\right), \widehat{C}=\left(\begin{array}{l}0 \\ I\end{array}\right)$, and the system (4) becomes in the following compact form:

$$
\begin{gathered}
\dot{X}=\widehat{A} X+F(X)+\widehat{B} G(X) \theta+D_{1}(t)+\widehat{C} D_{2}(t), \\
Y=z=\widehat{C}^{T} X,
\end{gathered}
$$

where $X \in R^{n+m}$ is the state vector and $Y \in R^{m}$ is the output vector of the system (5). $\widehat{A} \in R^{(n+m) \times(n+m)}, \widehat{B} \in R^{(n+m) \times p}$, and $\widehat{C} \in R^{(n+m) \times m}$ are constant matrices, and $(\widehat{A}, \widehat{C})$ is observable. $F: R^{n+m} \rightarrow R^{n+m}$ and $G: R^{n+m} \rightarrow R^{p \times q}$ are nonlinear functions and satisfy Lipschitz conditions:

$$
\begin{gathered}
\|F(X)-F(\widehat{X})\| \leq k_{F}\|X-\widehat{X}\|, \\
\|G(X)-G(\widehat{X})\| \leq k_{G}\|X-\widehat{X}\|,
\end{gathered}
$$

where $k_{F}$ and $k_{G}$ are Lipschitz constants.

Now, it treats (5) as the drive system, and the response system is

$$
\dot{\vec{X}}=\widehat{A} \widehat{X}+F(\widehat{X})+\widehat{B} G(\widehat{X}) \widehat{\theta}+L\left(Y-\widehat{C}^{T} \widehat{X}\right)+\widehat{B} u,
$$

where $u$ is nonlinear input and $L \in R^{n+m}$ is the undetermined gain matrix.

Similarly, if we define the synchronization error between the drive (5) and response systems (7) as $E=X-\widehat{X}$, the error system is gotten as follows:

$$
\begin{aligned}
\dot{E}= & \left(\widehat{A}-L \widehat{C}^{T}\right) E+F(X)-F(\widehat{X})+\widehat{B}(G(X) \theta-G(\widehat{X}) \widehat{\theta}) \\
& +D_{1}(t)+\widehat{C} D_{2}(t)-\widehat{B} u .
\end{aligned}
$$

From (8), it is seen that the external perturbations $D_{1}(t)$ and $\widehat{C} D_{2}(t)$ are all not relevant to the gain matrix $L$ if we choose $Y=z=\int_{0}^{t} y(\xi) d \xi$ as a new output vector of the system (5). Thus, to make the error system approach zero as a limit, the appropriate gain $L$ and the nonlinear input $u$ can be obtained according to the following scheme.

\section{Design of Adaptive Integral Observer}

In order to design an observer to realize synchronization between the master and slave systems, the following assumptions must be made. 
Assumption 1. The unknown parameter $\theta$ and external perturbations $D_{1}(t), D_{2}(t)$ of the system (5) are bounded, which satisfy $\|\theta\| \leq k_{\theta},\left\|D_{1}(t)\right\| \leq k_{D_{1}}$, and $\left\|D_{2}(t)\right\| \leq k_{D_{2}}$, where $k_{\theta}$, $k_{D_{1}}, k_{D_{2}}$ are unknown.

Assumption 2. The gain matrix $L$ satisfies the following conditions:

$$
\begin{gathered}
\left(\widehat{A}-L \widehat{C}^{T}\right)^{T} P+P\left(\widehat{A}-L \widehat{C}^{T}\right)=-Q, \\
\widehat{B}^{T} P=\widehat{C}^{T},
\end{gathered}
$$

where $P \in R^{(n+m) \times(n+m)}, Q \in R^{(n+m) \times(n+m)}$ are positive matrices.

Theorem 3. If the drive system (5) and the response system (7) satisfy Assumptions 1 and 2, the nonlinear input $u$ is described as

$$
u=\widehat{\gamma} \operatorname{sgn}\left(Y-\widehat{C}^{T} \widehat{X}\right)
$$

where the adaptive laws for the parameter $\widehat{\gamma}$ are

$$
\dot{\hat{\gamma}}=\delta\left\|Y-\widehat{C}^{T} \widehat{X}\right\|
$$

where the parameter $\delta$ is an arbitrary positive constant. The unknown parameters of the system (7) can be updated as follows:

$$
\dot{\hat{\theta}}=G^{T}(\widehat{X})\left(Y-\widehat{C}^{T} \widehat{X}\right) .
$$

Then, as time goes by, the drive system (5) with unknown parameters and external perturbations can synchronize the response system (7) from the arbitrary initial conditions.

Proof. Consider the following Lyapunov function candidate as

$$
V=E^{T} P E+(\theta-\widehat{\theta})^{T}(\theta-\widehat{\theta})+\frac{1}{\delta}(\gamma-\widehat{\gamma})^{2},
$$

where the constant $\gamma=\sigma k_{E} k_{F}+k_{E} k_{G} k_{\theta}+\sigma k_{D_{1}}+\sigma k_{\widehat{C}} k_{D_{2}}$.

The derivative of $V$ along the trajectories of the error system (8) is

$$
\begin{aligned}
\dot{V}= & \dot{E}^{T} P E+E^{T} P \dot{E}-2(\theta-\widehat{\theta})^{T} \dot{\hat{\theta}}-\frac{2}{\delta}(\gamma-\widehat{\gamma}) \dot{\hat{\gamma}} \\
= & -E^{T} Q E+2 E^{T} P(F(X)-F(\widehat{X})) \\
& +2 E^{T} P \widehat{B}(G(X)-G(\widehat{X})) \theta+2 E^{T} P \widehat{B} G(\widehat{X})(\theta-\widehat{\theta}) \\
& +2 E^{T} P D_{1}(t)+2 E^{T} P \widehat{C} D_{2}(t)-2 \widehat{\gamma}\left\|E^{T} P \widehat{B}\right\| \\
& -2(\theta-\widehat{\theta})^{T} G^{T}(\widehat{X}) \widehat{B}^{T} P E-2(\gamma-\widehat{\gamma})\left\|\widehat{B}^{T} P E\right\| \\
= & -E^{T} Q E+2 E^{T} P(F(X)-F(\widehat{X})) \\
& +2 E^{T} P \widehat{B}(G(X)-G(\widehat{X})) \theta \\
& +2 E^{T} P D_{1}(t)+2 E^{T} P \widehat{C} D_{2}(t)-2 \gamma\left\|\widehat{B}^{T} P E\right\|
\end{aligned}
$$

$$
\begin{aligned}
\leq & -E^{T} Q E+2\left\|E^{T} P(F(X)-F(\widehat{X}))\right\| \\
& +2\left\|E^{T} P \widehat{B}\right\|\|G(X)-G(\widehat{X})\|\|\theta\| \\
& +2\left\|E^{T} P D_{1}(t)\right\|+2\left\|E^{T} P \widehat{C} D_{2}(t)\right\|-2 \gamma\left\|\widehat{B}^{T} P E\right\| .
\end{aligned}
$$

As we know, the states of the dynamical systems are always bounded when they are periodic, chaotic or equilibrium. So, for the state vector of the systems (5), (7), and (8), they satisfy the following inequalities:

$$
\begin{aligned}
\|X(t)\| & \leq k_{X}, \quad \forall t \in[0, \infty), k_{X} \in R^{+}, \\
\|\widehat{X}(t)\| & \leq k_{\widehat{X}}, \quad \forall t \in[0, \infty), k_{\widehat{X}} \in R^{+}, \\
\|E(t)\| & =\|X(t)-\widehat{X}(t)\| \\
& \leq k_{X}+k_{\widehat{X}}=k_{E}, \quad \forall t \in[0, \infty), k_{E} \in R^{+} .
\end{aligned}
$$

Let the constants $\sigma=\left\|\left[\left(\widehat{B} \widehat{B}^{T}\right)^{-1}\right]^{T} \widehat{B}\right\|, \quad k_{\widehat{C}}=\|\widehat{C}\|$; we can derive

$$
\begin{aligned}
& \left\|E^{T} P(F(X)-F(\widehat{X}))\right\| \\
& \quad\left\|E^{T} P \widehat{B} \widehat{B}^{T}\left(\widehat{B} \widehat{B}^{T}\right)^{-1}(F(X)-F(\widehat{X}))\right\| \\
& \leq\left\|E^{T} P \widehat{B}\right\|\left\|\widehat{B}^{T}\left(\widehat{B} \widehat{B}^{T}\right)^{-1}\right\|\|F(X)-F(\widehat{X})\| \\
& \quad \leq \sigma k_{E} k_{F}\left\|E^{T} P \widehat{B}\right\|, \\
& \|G(X)-G(\widehat{X})\|\|\theta\| \leq k_{G}\|E\|\|\theta\| \leq k_{E} k_{G} k_{\theta}, \\
& \left\|E^{T} P D_{1}(t)\right\|=\left\|E^{T} P \widehat{B} \widehat{B}^{T}\left(\widehat{B} \widehat{B}^{T}\right)^{-1} D_{1}(t)\right\| \leq \sigma k_{D_{1}}\left\|E^{T} P \widehat{B}\right\|, \\
& \left\|E^{T} P \widehat{C} D_{2}(t)\right\| \leq \sigma k_{\widehat{C}} k_{D_{2}}\left\|E^{T} P \widehat{B}\right\| .
\end{aligned}
$$

So the derivative of $V$ is further gotten

$$
\begin{aligned}
\dot{V} \leq & -E^{T} Q E+2\left\|E^{T} P(F(X)-F(\widehat{X}))\right\| \\
& +2\left\|E^{T} P \widehat{B}\right\|\|G(X)-G(\widehat{X})\|\|\theta\| \\
& +2\left\|E^{T} P D_{1}(t)\right\|+2\left\|E^{T} P \widehat{C} D_{2}(t)\right\|-2 \gamma\left\|\widehat{B}^{T} P E\right\| \\
\leq & -E^{T} Q E+2 \sigma k_{E} k_{F}\left\|E^{T} P \widehat{B}\right\|+2 k_{E} k_{G} k_{\theta}\left\|E^{T} P \widehat{B}\right\| \\
& +2 \sigma k_{D_{1}}\left\|E^{T} P \widehat{B}\right\| \\
& +2 \sigma k_{\widehat{C}} k_{D_{2}}\left\|E^{T} P \widehat{B}\right\|-2 \gamma\left\|\widehat{B}^{T} P E\right\| .
\end{aligned}
$$

Since the parameter $\gamma=\sigma k_{E} k_{F}+k_{E} k_{G} k_{\theta}+\sigma k_{D_{1}}+\sigma k_{\widehat{C}} k_{D_{2}}$, then it yields

$$
\dot{V} \leq-E^{T} Q E \leq-\lambda_{\min }(Q)\|E\|^{2}=-\omega(t) .
$$


Integration (18) from 0 to $t$, it gains the following inequality:

$$
V(0) \geq V(t)+\int_{0}^{t} \omega(s) d s \geq \int_{0}^{t} \omega(s) d s .
$$

As $t \rightarrow \infty$, and $V(0)$ is positive and bounded, $\lim _{t \rightarrow \infty} \int_{0}^{t} \omega(s) d s$ exists and is bounded. Then according to Barbalat lemma, it obtains

$$
\lim _{t \rightarrow \infty} \omega(t)=\lambda_{\min }(Q) \lim _{t \rightarrow \infty}\|E(t)\|^{2}=0
$$

It implies that the error vector of the system (8) asymptotically approaches zero as time goes by and chaos synchronization is realized successfully by using the designed adaptive observer. Thus, the proof is complete.

Remark 4. We say that this approach has fewer constraints because of the following reasons. According to the above discussion, we have seen that, for the bounds of external perturbations $k_{D_{1}}$ and $k_{D_{2}}$, the bound of the unknown parameter $k_{\theta}$, the Lipchitz constants $k_{F}$ and $k_{G}$, and the bound of the error vector $k_{E}$, the parameters $\sigma, k_{\hat{C}}$, all these constants are only used in proof, and even if these constants are unknown, the adaptive observer can still be used to realize synchronization based on Theorem 3 .

Remark 5. Theorem 3 is also suitable to those chaotic systems with known parameters and external perturbations only if treating the nonlinear function $G(X)=0$, which is also verified in the following example.

Remark 6. According to Kalman-Yakubovich-Popov lemma [17], for the response system (7), the gain matrix $L$ can be chosen such that the transfer function matrix

$$
H(s)=\widehat{C}\left(s I_{n}-(\widehat{A}-L \widehat{C})\right)^{-1} \widehat{B}
$$

is strictly positive real, then there exits matrices $P \in R^{n \times n}$, $Q \in R^{n \times n}$ satisfying Assumption 2 .

\section{Numerical Simulation}

4.1. Chaos Synchronization of Rössler System. Firstly, we choose the perturbed Rössler system with known parameters, that is, $G(X)=0$, to illustrate the effectiveness of the proposed scheme. The Rössler is described by

$$
\begin{aligned}
& \dot{x}_{1}=-x_{2}-x_{3}, \\
& \dot{x}_{2}=x_{1}+a x_{2}, \\
& \dot{x}_{3}=c+x_{3}\left(x_{1}-b\right) .
\end{aligned}
$$

If the parameters are chosen as $a=0.2, b=5.7, c=$ 0.2 , the system shows chaotic behavior. Figure 1 gives the attractors of Rössler system (22) and time evolution of three states of the system (22) is shown in Figure 2.
When the original system is affected by external disturbances, the system becomes

$$
\begin{gathered}
\left(\begin{array}{c}
\dot{x}_{1} \\
\dot{x}_{2} \\
\dot{x}_{3}
\end{array}\right)=\left(\begin{array}{ccc}
0 & -1 & -1 \\
1 & 0.2 & 0 \\
0 & 0 & -5.7
\end{array}\right)\left(\begin{array}{l}
x_{1} \\
x_{2} \\
x_{3}
\end{array}\right)+\left(\begin{array}{c}
0 \\
0 \\
x_{1} x_{3}
\end{array}\right)+\left(\begin{array}{l}
d_{1}(t) \\
d_{2}(t) \\
d_{3}(t)
\end{array}\right), \\
y=x_{1}=\left(\begin{array}{lll}
1 & 0 & 0
\end{array}\right) x+D_{2}(t)
\end{gathered}
$$

where $D_{2}(t)$ and $d_{i}(t), i=1,2,3$ are external perturbations.

In order to use the proposed method in this paper, the system (23) is rewritten in the following compact form

$$
\begin{gathered}
\dot{X}=\widehat{A} X+F(X)+D_{1}(t)+\widehat{C} D_{2}(t), \\
Y=z=\widehat{C}^{T} X
\end{gathered}
$$

where the state $X=\left(\begin{array}{llll}x_{1} & x_{2} & x_{3} & z\end{array}\right)^{T}$, the nonlinear function $F(X)=\left(\begin{array}{llll}0 & 0 & x_{1} x_{3} & 0\end{array}\right)^{T}$, the external perturbation $D_{1}(t)=$ $\left(\begin{array}{llll}d_{1}(t) & d_{2}(t) & d_{3}(t) & 0\end{array}\right)^{T}$, the constant matrix $\widehat{C}=\left(\begin{array}{llll}0 & 0 & 0 & 1\end{array}\right)^{T}$, and the matrix $\widehat{A}$ is

$$
\widehat{A}=\left(\begin{array}{cccc}
0 & -1 & -1 & 0 \\
1 & 0.2 & 0 & 0 \\
0 & 0 & -5.7 & 0 \\
1 & 0 & 0 & 0
\end{array}\right)
$$

One can get that $\left(\widehat{A}, \widehat{C}^{T}\right)$ is observable and the output of the system is $Y=z$. It regards the system (24) as the drive system, and the response system is

$$
\begin{aligned}
\left(\begin{array}{c}
\dot{\hat{x}}_{1} \\
\dot{\hat{x}}_{2} \\
\dot{\hat{x}}_{3} \\
\dot{\vec{z}}
\end{array}\right)= & \left(\begin{array}{cccc}
0 & -1 & -1 & 0 \\
1 & 0.2 & 0 & 0 \\
0 & 0 & -5.7 & 0 \\
1 & 0 & 0 & 0
\end{array}\right)\left(\begin{array}{c}
\widehat{x}_{1} \\
\widehat{x}_{2} \\
\widehat{x}_{3} \\
\widehat{z}
\end{array}\right)+\left(\begin{array}{c}
0 \\
0 \\
x_{1} x_{3} \\
0
\end{array}\right) \\
& +L(z-\widehat{z})+\widehat{B} u
\end{aligned}
$$

where the vector $\widehat{B}=\left(\begin{array}{llll}1 & 0 & 1 & 1\end{array}\right)^{T}$.

In numerical simulations, the external perturbations of the drive system $(24)$ are chosen $D_{1}(t)=(0.028 \cos 3 t$ $0.019 \sin 7 t 0.034 \sin 6 t 0)^{T}$ and $D_{2}(t)=0.012 \cos 10 t$, respectively. The gain matrix is $L=(19.9044-8.684680 .9513$ $0.5940)^{T}$, it can get and that the eigenvalues of $\widehat{A}-L \widehat{C}^{T}$ are $-1.38 \pm 2.491 i$, and $-1.667 \pm 1.715 i$, the transfer function is obtained as follows:

$$
\begin{aligned}
H(s) & =\widetilde{C}^{T}\left(s I-\left(\widetilde{A}-L \widetilde{C}^{T}\right)\right)^{-1} \widetilde{B} \\
& =\frac{s^{3}+6.5 s^{2}+4.36 s+4.76}{s^{4}+6.094 s^{3}+23.03 s^{2}+42.82 s+46.39},
\end{aligned}
$$

which is strictly positive real.

It is chosen that the initial values of the drive system (24) and response system $(26)$ are $\left(x_{1}(0) x_{2}(0) x_{3}(0) z(0)\right)^{T}=$ $\left(\begin{array}{llll}-1 & 1 & 0 & 2\end{array}\right)^{T}$ and $\left(\widehat{x}_{1}(0) \widehat{x}_{2}(0) \widehat{x}_{3}(0) \widehat{z}(0)\right)^{T}=\left(\begin{array}{llll}-3 & 1 & 1 & 3.2\end{array}\right)^{T}$. 


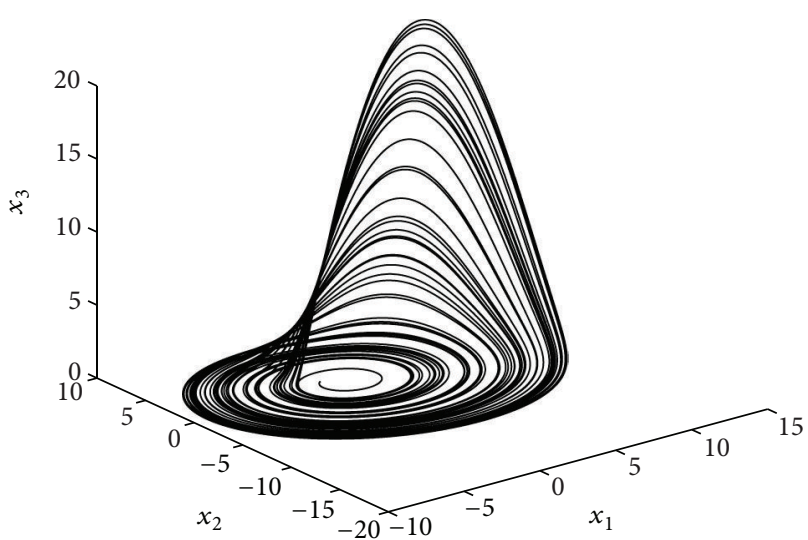

(a)

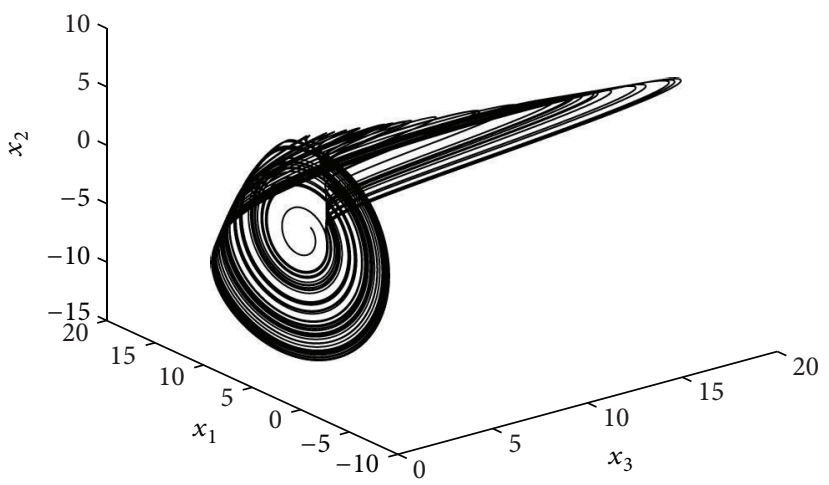

(b)

FIGURE 1: The attractors of Rössler system (a) $x_{1}-x_{2}-x_{3}$ and (b) $x_{3}-x_{1}-x_{2}$.

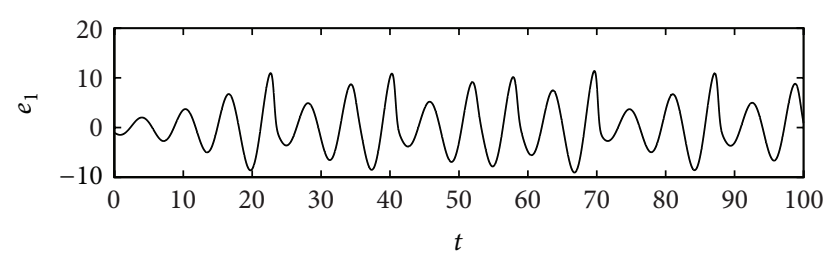

(a)

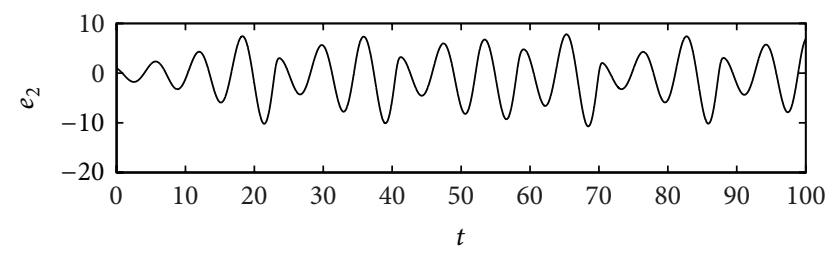

(b)

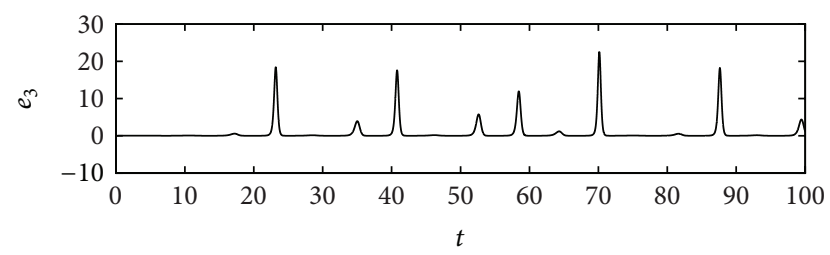

(c)

Figure 2: Time evolution of Rössler system.

The nonlinear input $u$ is given according to (10). The parameter $\delta$ is selected as 1.2 , and the initial value of $\widehat{\gamma}(0)$ is 0.1 . Figure 3 gives time evolution of the synchronization errors between the drive (24) and response systems (26) with the nonlinear input (10) and the gain matrix $L$. It shows that the error vector approaches zero asymptotically with time passage, so the drive system (24) with external perturbations and unknown parameters synchronizes the response system (26) by using the proposed method.

4.2. Chaos Synchronization of Cellular Neural Network System. We use cellular neural network (CNN) system with unknown

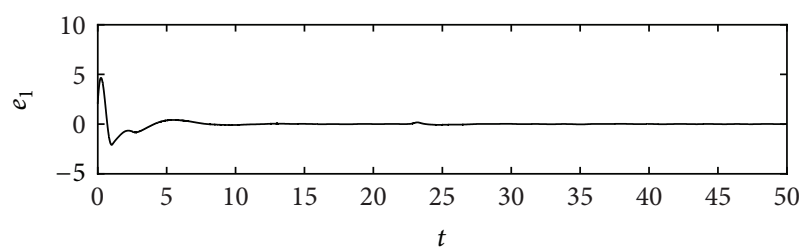

(a)

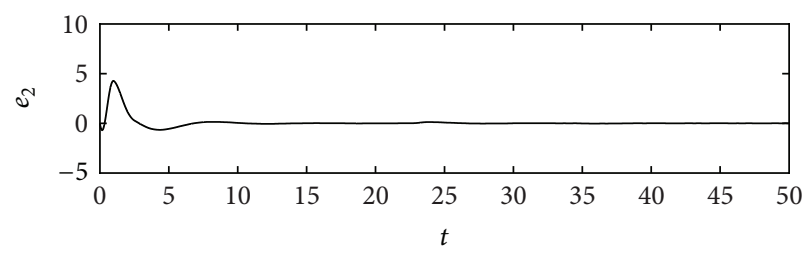

(b)

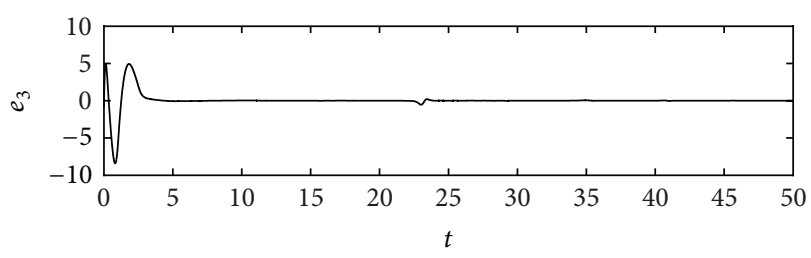

(c)

FIGURE 3: Time evolution of the synchronization errors of Rössler system with the gain matrix and nonlinear input.

parameters and external perturbations as another example to verify the effectiveness of the proposed scheme.

CNN system has aroused wide concern in private communication, image processing, and pattern recognition due to its characteristic of strong real time, complex dynamics, high computing speed, and so forth. The three-order CNN nonlinear system is

$$
\begin{gathered}
\dot{x}_{1}=a x_{1}+b x_{2}+\theta\left(\left|x_{1}+1\right|-\left|x_{1}-1\right|\right), \\
\dot{x}_{2}=x_{1}-x_{2}+x_{3}, \\
\dot{x}_{3}=c x_{2} .
\end{gathered}
$$




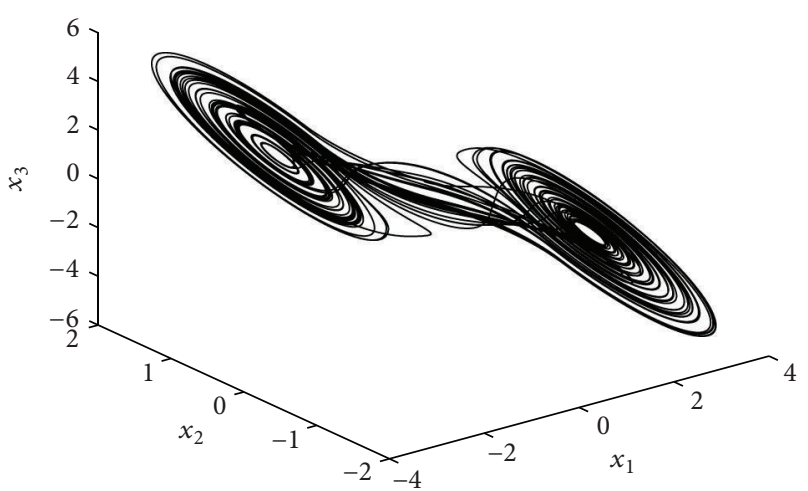

(a)

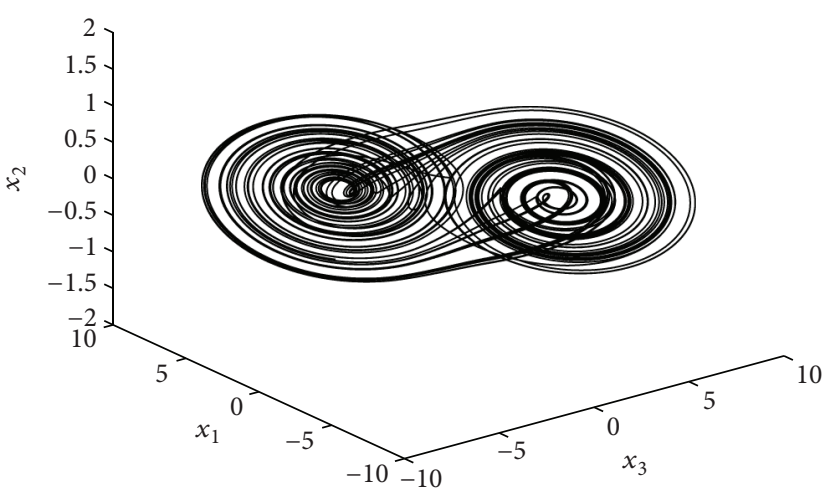

(b)

FIGURE 4: The attractors of CNN system (a) $x_{1}-x_{2}-x_{3}$ and (b) $x_{3}-x_{1}-x_{2}$.

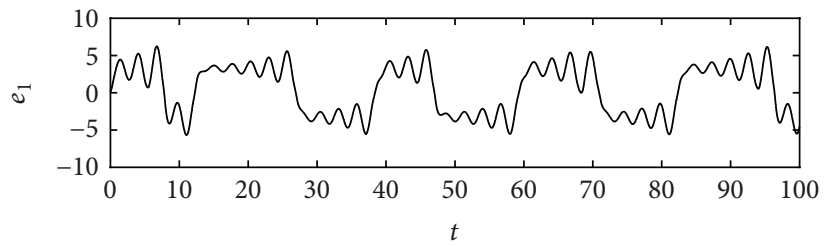

(a)

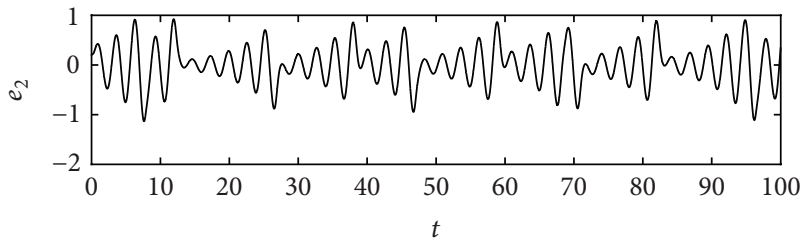

(b)

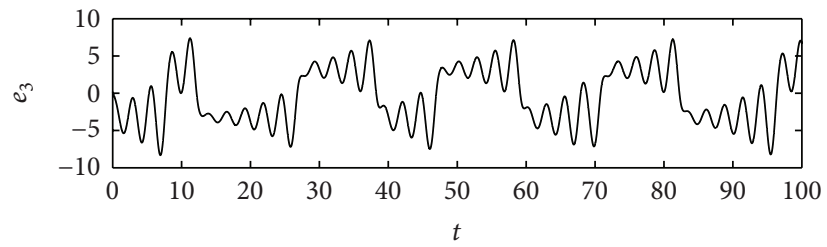

(c)

Figure 5: Time evolution of CNN system.

When the parameters $\theta=2, a=-1.2179, b=8.342$, and $c=-11.925$, this system has a chaotic attractor. Figure 4 gives the attractors of CNN system (28) and Figure 5 shows time evolution of its states.

If we regard $\theta$ as unknown parameter, the $\mathrm{CNN}$ chaotic system with external perturbations can be written as

$$
\begin{aligned}
\left(\begin{array}{l}
\dot{x}_{1} \\
\dot{x}_{2} \\
\dot{x}_{3}
\end{array}\right)= & \left(\begin{array}{ccc}
-1.2179 & 8.342 & 0 \\
1 & -1 & 1 \\
0 & -11.925 & 0
\end{array}\right)\left(\begin{array}{l}
x_{1} \\
x_{2} \\
x_{3}
\end{array}\right) \\
& +\left(\begin{array}{l}
1 \\
0 \\
0
\end{array}\right)\left(\left|x_{1}+1\right|-\left|x_{1}-1\right|\right) \theta+\left(\begin{array}{l}
d_{1}(t) \\
d_{2}(t) \\
d_{3}(t)
\end{array}\right), \\
y= & x_{1}=\left(\begin{array}{lll}
1 & 0 & 0
\end{array}\right) x+D_{2}(t),
\end{aligned}
$$

where $D_{2}(t)$ and $d_{i}(t), i=1,2,3$ are external perturbations.
In order to use adaptive integral observer to realize synchronization, the system (29) becomes of the following compact form

$$
\begin{gathered}
\dot{X}=\widehat{A} X+\widehat{B} G(X) \theta+D_{1}(t)+\widehat{C} D_{2}(t), \\
Y=z=\widehat{C}^{T} X,
\end{gathered}
$$

where the state vector $X=\left(\begin{array}{llll}x_{1} & x_{2} & x_{3} & z\end{array}\right)^{T}$, the nonlinear function $G(X)=\left|x_{1}+1\right|-\left|x_{1}-1\right|$, the matrices $\widehat{B}=$ $\left(\begin{array}{llll}1 & 0 & 0 & 0\end{array}\right)^{T}$ and $\widehat{C}=\left(\begin{array}{llll}0 & 0 & 0 & 1\end{array}\right)^{T}$, in addition, the external perturbation $D_{1}(t)=\left(\begin{array}{llll}d_{1}(t) & d_{2}(t) & d_{3}(t) & 0\end{array}\right)^{T}$, and the constant matrix

$$
\widehat{A}=\left(\begin{array}{cccc}
-1.2179 & 8.342 & 0 & 0 \\
1 & -1 & 1 & 0 \\
0 & -11.925 & 0 & 0 \\
1 & 0 & 0 & 0
\end{array}\right) .
$$

One can get that $\left(\widehat{A}, \widehat{C}^{T}\right)$ is observable. In simulation, we choose that the gain matrix is $L=(11.43191 .7566$ $-14.81054 .9021)^{T}$, and the external perturbations are $D_{1}(t)=$

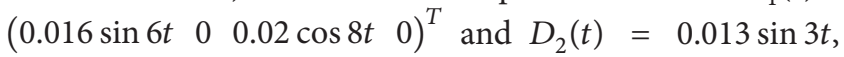




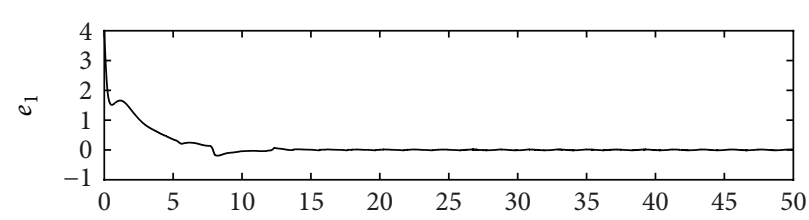

(a)

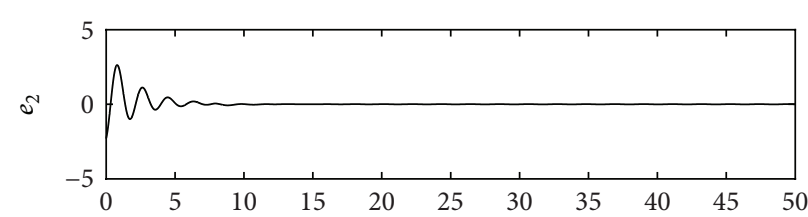

(b)

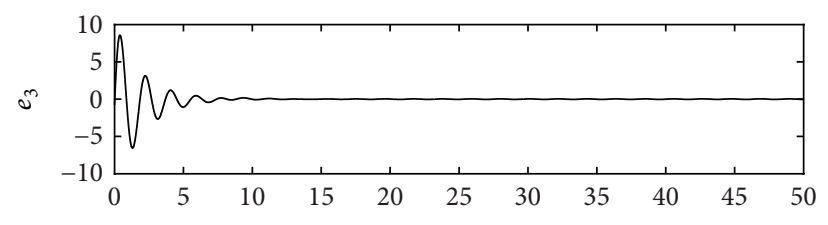

(c)

FIGURE 6: Time evolution of the synchronization errors of CNN system with the gain matrix and nonlinear input.

respectively. The initial values of the drive system and the response system are $\left(x_{1}(0) \quad x_{2}(0) \quad x_{3}(0) \quad z(0)\right)^{T}=\left(\begin{array}{lll}0.1 & 0.2\end{array}\right.$

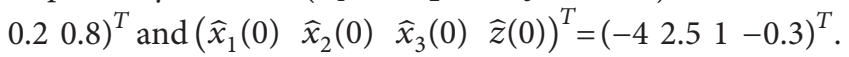
The parameter $\delta$ is selected as 0.1 , and the initial value of $\hat{\gamma}(0)$ is 0.3 . Figure 6 shows the time evolution of $\mathrm{CNN}$ error system. It can be seen that, with time passage, the synchronization error vector tends to zero. So the master system with unknown parameters and external perturbations synchronizes the slave system, which shows that our proposed method is effective.

\section{Conclusions}

In this paper, we design a new adaptive integral observer to realize chaos synchronization when both the state vector and the output of the original system are all perturbed for some reason. The proposed method is not only suitable to such chaotic systems with known parameters but also applies to such chaotic systems with unknown parameters. We can see that this method has fewer constraints, so it is easy to applied in practice. In the end, numerical simulations are illustrated to further verify the validity of the approach.

\section{Acknowledgment}

This project is supported by the National Natural Science Foundation of China (Grant nos. 11002114, 60901076, 11102157, 11202035 , and 11272258).

\section{References}

[1] L. M. Pecora and T. L. Carroll, "Synchronization in chaotic systems," Physical Review Letters, vol. 64, no. 8, pp. 821-824, 1990.

[2] L. Kocarev and U. Parlitz, "General approach for chaotic synchronization with applications to communication," Physical Review Letters, vol. 74, no. 25, pp. 5028-5031, 1995.

[3] D. Huang, "Simple adaptive-feedback controller for identical chaos synchronization," Physical Review E, vol. 71, no. 3, Article ID 037203, 2005.
[4] H. Salarieh and A. Alasty, "Adaptive chaos synchronization in Chua's systems with noisy parameters," Mathematics and Computers in Simulation, vol. 79, no. 3, pp. 233-241, 2008.

[5] T. Wang and N. Jia, "Chaos control and hybrid projective synchronization of several new chaotic systems," Applied Mathematics and Computation, vol. 218, no. 13, pp. 7231-7240, 2012.

[6] X. Yang, C. Huang, and Q. Zhu, "Synchronization of switched neural networks with mixed delays via impulsive control," Chaos, Solitons \& Fractals, vol. 44, no. 10, pp. 817-826, 2011.

[7] Y.-M. Li and Y.-Y. Sun, "Type-2 T-S fuzzy impulsive control of nonlinear systems," Applied Mathematical Modelling, vol. 36, no. 6, pp. 2710-2723, 2012.

[8] S. Bowong, F. M. M. Kakmeni, and C. Tchawoua, "Controlled synchronization of chaotic systems with uncertainties via a sliding mode control design," Physical Review E, vol. 70, no. 6, Article ID 066217, 2004.

[9] M. P. Aghababa and A. Heydari, "Chaos synchronization between two different chaotic systems with uncertainties, external disturbances, unknown parameters and input nonlinearities," Applied Mathematical Modelling, vol. 36, no. 4, pp. 16391652, 2012.

[10] Y.-C. Hung, J.-J. Yan, and T.-L. Liao, "Projective synchronization of Chua's chaotic systems with dead-zone in the control input," Mathematics and Computers in Simulation, vol. 77, no. 4, pp. 374-382, 2008.

[11] X.-Y. Wang and B. Fan, "Generalized projective synchronization of a class of hyperchaotic systems based on state observer," Communications in Nonlinear Science and Numerical Simulation, vol. 17, no. 2, pp. 953-963, 2012.

[12] D. M. Senejohnny and H. Delavari, "Active sliding observer scheme based fractional chaos synchronization," Communications in Nonlinear Science and Numerical Simulation, vol. 17, no. 11, pp. 4373-4383, 2012.

[13] C. Hua, X. Guan, X. Li, and P. Shi, "Adaptive observer-based control for a class of chaotic systems," Chaos, Solitons \& Fractals, vol. 22, no. 1, pp. 103-110, 2004.

[14] S. Bowong, F. M. Moukam Kakmeni, and H. Fotsin, "A new adaptive observer-based synchronization scheme for private communication," Physics Letters A, vol. 355, no. 3, pp. 193-201, 2006.

[15] F. Chen and W. Zhang, "LMI criteria for robust chaos synchronization of a class of chaotic systems," Nonlinear Analysis. Theory, Methods \& Applications A, vol. 67, no. 12, pp. 3384-3393, 2007. 
[16] P. Mahdi and J. M. Vahid, "A novel robust proportional-integral (PI) adaptive observer design for chaos synchronization," Chinese Physics B, vol. 20, Article ID 120503, 2011.

[17] F. Zhu, "Full-order and reduced-order observer-based synchronization for chaotic systems with unknown disturbances and parameters," Physics Letters A, vol. 372, no. 3, pp. 223-232, 2008.

[18] Y. Wang, X. Xu, L. Dai, and B. Hou, "Synchronization of a class of partially unknown chaotic systems with integral observer," in Proceedings of the 36th Annual Conference of the IEEE Industrial Electronics Society (IECON '10), pp. 231-235, November 2010. 


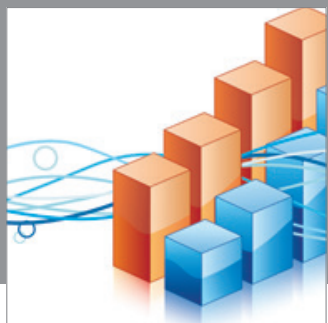

Advances in

Operations Research

mansans

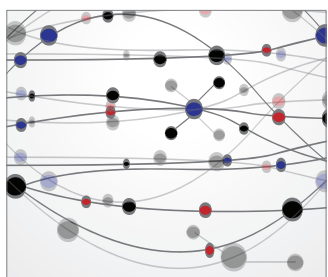

The Scientific World Journal
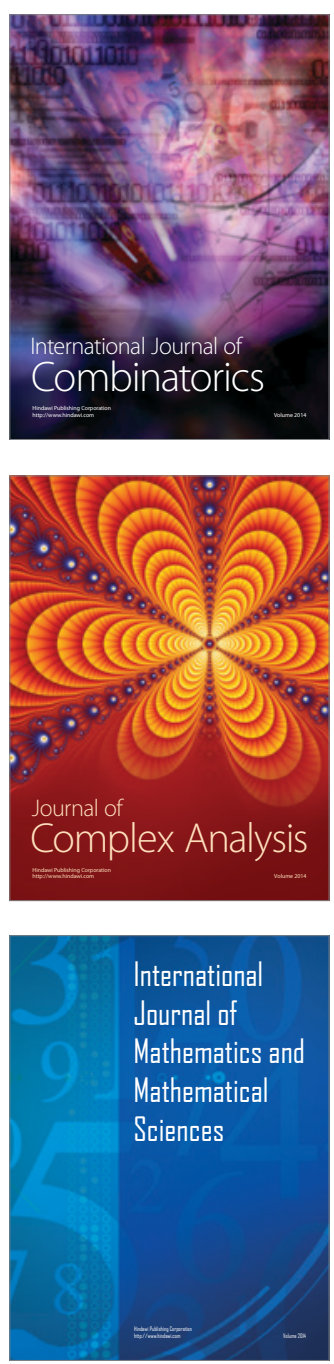
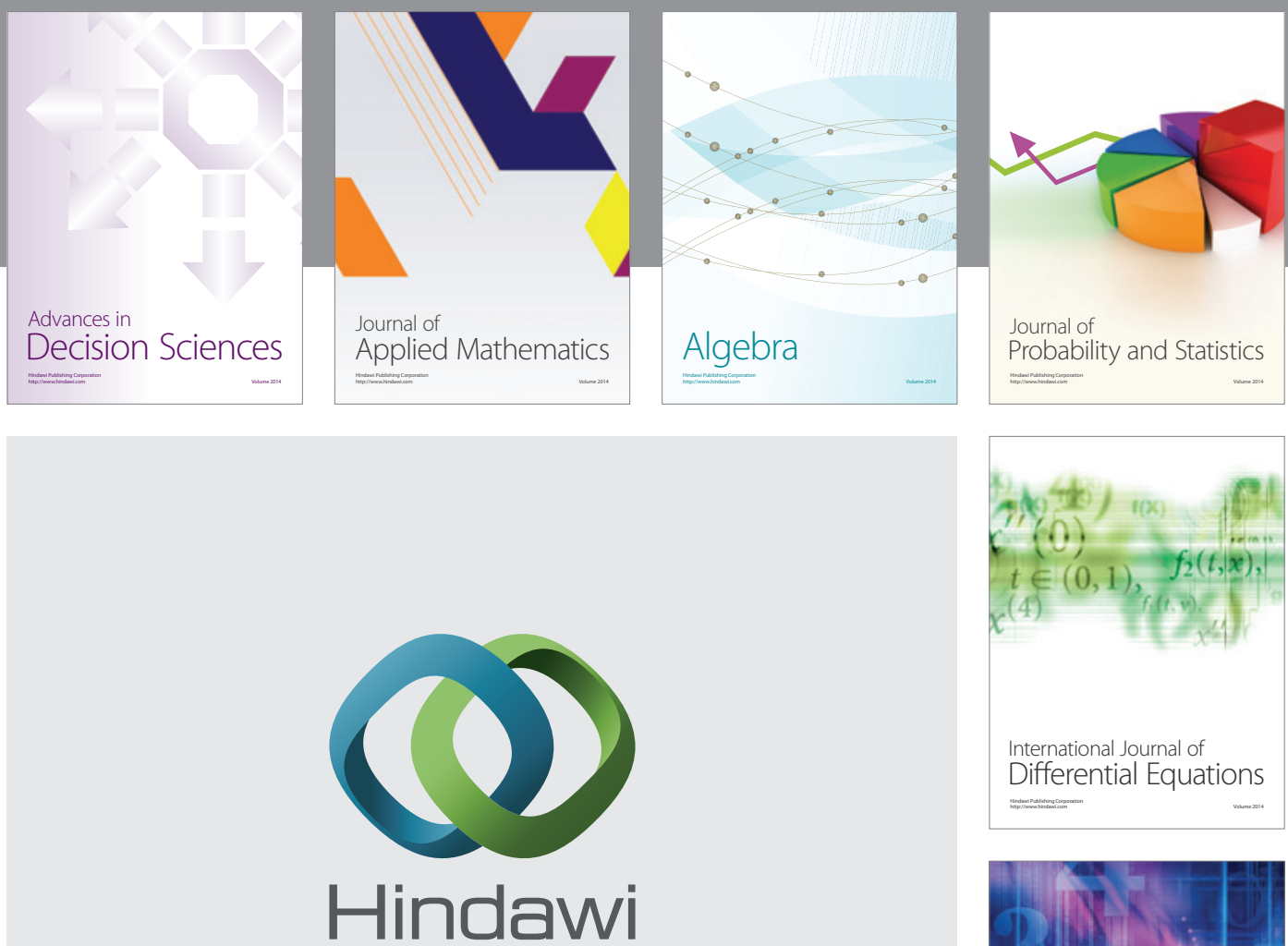

Submit your manuscripts at http://www.hindawi.com
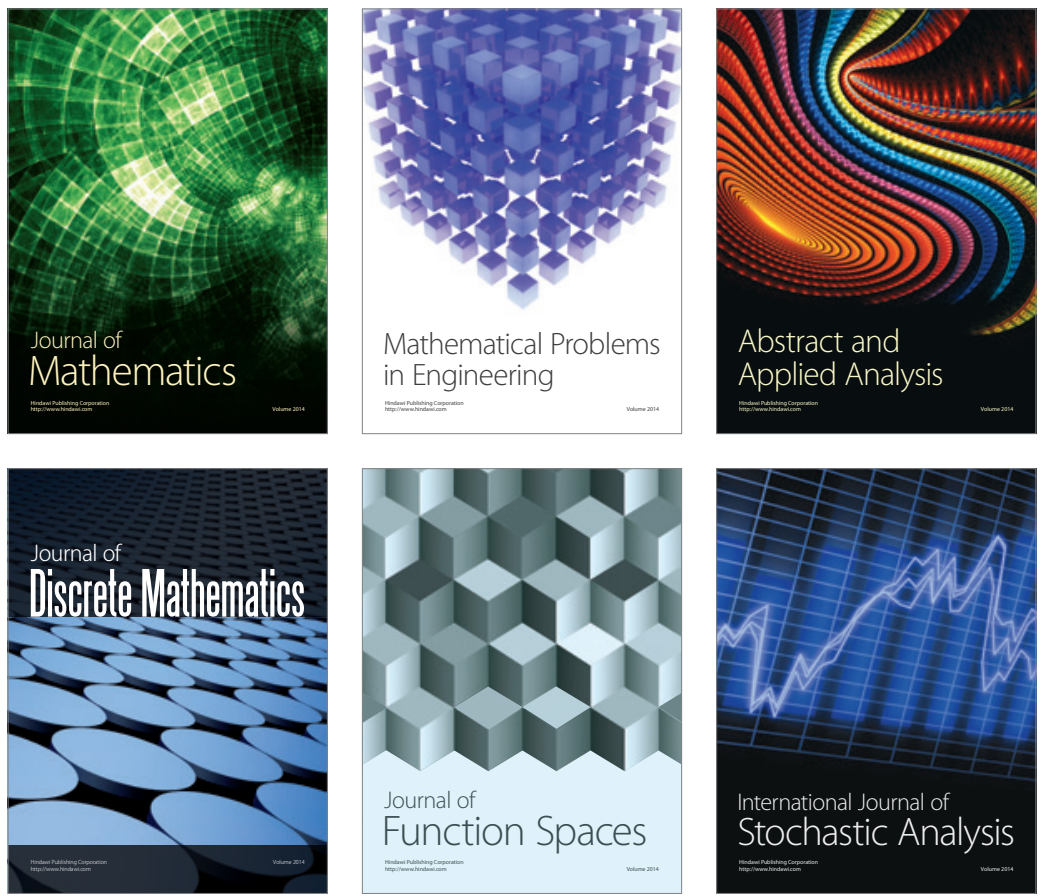

Journal of

Function Spaces

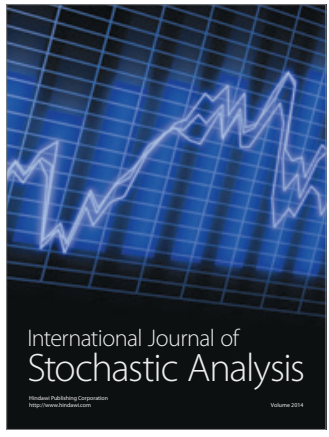

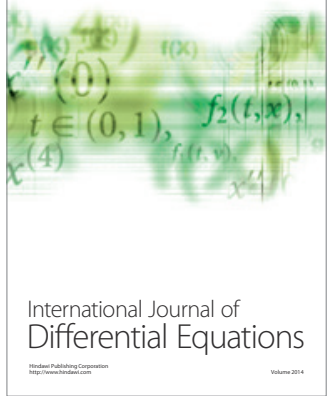
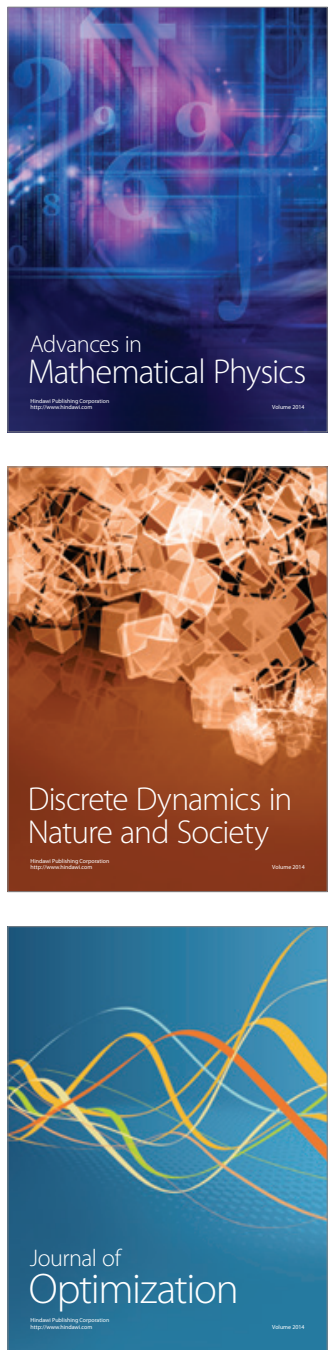